measles-vaccine-associated cases. Only three of the 11 proved abnormal (dead, or with neurological, educational, or behavioural dysfunction), for whom there were seven matched controls defined as before (RR $0.84 ; 95 \%$ CI $0 \cdot 20-3 \cdot 49$ ). Although the number of vaccine-associated cases is small, these findings provide no evidence of a risk of long-term neurological damage associated with measles vaccine.

* David Miller, Jane Wadsworth, Judith Diamond, Euan Ross

*Department of Eprdemology and Public Health, Imperial College School of Medicine at St Many's London W2 1PG, UK; and Department of Community Paediatrics, King's College Hospital School of Medicine and Dentistry, London

1 da Silverra $C M$, Salisbury DM, de Quadros CA. Measles vaccination and Guillan-Barré syndrome. Lancet 1997; 349: 14-16.

2 Wing L. Autism spectrum disorders: no evidence for or against an increase in prevalence. BM7 1996; 312: 327-28.

3 Miller DL, Ross EM, Alderslade R, Bellman MH, Rawson NSB. Pertussis immunisation and serious acute neurological llness in children. BMF 1981; 282: 1595-99.

4 Miller D, Madge N, Diamond J, Wadsworth J, Ross E. Pertussis immunisation and serious acute neurological illnesses in children. BMJ 1993; 307: 1171-76.

\section{Third-generation oral contraceptives and venous thrombosis}

SIR-Farmer and colleagues' show again that third-generation contraceptives have a higher risk of venous thrombosis (VTE) (a significant relative risk of 1.7 ), in a non-research database without any attempt at objective diagnosis. Since clinical diagnoses of VTE are wrong more than half the time, ${ }^{2}$ this will very strongly dilute the findings. Whether Farmer's report deals with first thrombotic episodes is debatable. The main message is that the data are very resistant: only in sub-subgroup analyses or in an over-adjusted logistic model, does the relative risk (almost) disappear.

When the hypothesis of confounding by prescribing is proposed, the analysis should focus on risk factors for venous thrombosis that are present at the time of prescription in healthy young women: age, body mass index, and familial occurrence. Age was adjusted in 5-year age groups in the cohort analysis; a true demonstration of the importance of age adjustment would be to show that adjustment by single year of age on the same data would make the association disappear-that demonstration is not reported, however. In fact, the adjusted rate ratio $(1 \cdot 7)$ is the same as the crude ratio $(1 \cdot 6)$. No information is presented on familial history. By contrast, unnecessary variables were entered in the model; some that are certain to be correlated with oral-contraceptive exposure, such as months of use, and others that will render the model unstable because they apply only to a few persons, such as "previous use of Schering PC4", or because they are correlated with the type of contraceptive, such as "recent change of pills".

The finding of the highest relative risk in users of a $20 \mu \mathrm{g}$ ethinyloestradiol third-generation pill can make perfect sense: it is a combination of a starter effect and a third-generation effect. During the period of the study the 20 $\mu \mathrm{g}$ ethinyloestradiol preparation was predominantly prescribed to new users, and had not been in use for long. WHO data have shown that in the first year of use, new users have the highest relative risks. ${ }^{3}$ If they use a third-generation contraceptive their risk doubles again. That the increased risk of thirdgeneration contraceptives remains, even with a low dose of oestrogens, should not come as a surprise: even the very low doses of oestrogens as are used in hormonal replacement therapy have almost the same relative risk of venous thrombosis as oral contraceptives.' If such a low dose of oestrogens is coupled to a third-generation progestagen that further increases its thrombogenic potential, and if this is preferentially prescribed to new users, we would expect the effect found by Farmer and co-workers.

Farmer and colleagues' report contains two gross misreadings of our previous publication. They imply that no previous studies applied sufficiently fine age-stratification, and that we did not make the distinction between 20 and $30 \mu \mathrm{g}$ ethinyloestradiol preparations. In the Leiden study we adjusted by single year of age, which is as adequate as age-matching with a variable case/control ratio, and as stated explicitely in the text and in the table, there were no users of $20 \mu \mathrm{g}$ ethinyloestradiol preparations. Nevertheless, the same two-fold increase in risk was found.

\section{*Jan P Vandenbroucke,}

Frans $M$ Helmerhorst.

Kitty W M Bloemenkamp.

\section{Frits $R$ Rosendaal}

Departments of $*$ Clinical Epıdemıology, Obstetrics, Gynecology and Reproductive Medicine, and Thrombosis and Haemostasıs Research Centre, Leiden University Hospital. PO Box 9600, 2300 RC Lejden. Netherlands

Farmer RDT, Lawrenson RA,

Thompson CR, Kennedy JG,

Hambleton IR. Population-based study of risk of venous thrombeembolism associated with oral contraceptives. Lancet $1997 ; 349$ : 83-88.

2 Prins $M H$, Turpie AGG. Diagnosis and treatment of venous thromboembolism. In: Bloom, eds. Haemostasis and thrombosis, 3rd ed. Edinburgh: Churchill-Livingstone 1994: 1381

3 Farley TMM, Merik O, Poulter NR, Chang CL, Marmot MG. Oral contraceptives and thrombotic diseases: impact of new epidemiologic studies. Contraception 1996; 54: 193-95.

4 Vandenbroucke JP, Helmerhorst FM. Risk of venous thrombosis with hormonereplacement therapy. Lancet 1996; 348: 972.

SIR-It is fundamental and indeed should be self-evident that in an observational drug safety study the people with the illness of interest (ie, the cases) must, in fact, have had the illness under study. In addition, to invoke a possible causal connection to the drug, individuals included as cases should have no other demonstrable proximate cause of the study illness-ie, they should reflect idiopathic disease. These principles are especially relevant when venous thromboembolism (VTE) is the outcome illness since (1) a firm diagnosis of VTE is uncertain in the absence of diagnostic tests for confirmation, and (2) there are many non-drug causes of VTE, such as trauma, recent surgery. Unless one examines clinical records, it is impossible to ascertain whether a case of VTE has been documented by diagnostic tests (ie, whether it is in fact a case), nor is it possible to establish with confidence whether it is idiopathic.

Farmer and colleagues" study relies solely on a general-practice, computerrecorded diagnosis of VTE together with a notation of anticoagulant therapy to define the cases. Such case inclusion is unsatisfactory since it surely includes cases (misdiagnosed) that in fact are not cases, as well as cases with a proximate cause such as trauma in which no drug effect can be reasonably expected. Computer review is inadequate to identify such individuals.

We have published the results of several studies relating oral contraceptives to idiopathic VTE. ${ }^{2,3}$ In all, whether interview based or computer based, we have excluded for reasons noted above more than $50 \%$ of potential cases from the final case series after review of the clinical records. In view of the failure to document and include only relevant cases, we deem the information provided by Farmer and colleagues to be uninterpretable.

Farmer and colleagues invoke strong residual confounding by age to explain the findings of our study ${ }^{3}$ and others recently reported." ${ }^{\prime 3}$ Their reference to the age adjustment in our study was incomplete and misleading. In fact, controls were matched to cases within 2 years in $85 \%$ of instances and the mean 